PDES, SUBMANIFOLDS AND

AFFINE DIFFERENTIAL GEOMETRY

BANACH CENTER PUBLICATIONS, VOLUME 69

INSTITUTE OF MATHEMATICS

POLISH ACADEMY OF SCIENCES

WARSZAWA 2005

\title{
CURVATURE CONDITIONS ON HYPERSURFACES WITH TWO DISTINCT PRINCIPAL CURVATURES
}

\author{
MAŁGORZATA GEOGOWSKA \\ Department of Mathematics, Agricultural University of Wrockaw \\ Grunwaldzka 53, 50-357 Wrockaw, Poland \\ E-mail: mglog@ozi.ar.wroc.pl
}

\begin{abstract}
We investigate curvature properties of hypersurfaces in semi-Riemannian spaces of constant curvature with the minimal polynomial of the second fundamental tensor of second degree. We present suitable examples of hypersurfaces.
\end{abstract}

1. Introduction. A semi-Riemannian manifold $(M, g), n=\operatorname{dim} M \geq 3$, is said to be an Einstein manifold if

$$
S=\frac{\kappa}{n} g
$$

on $M$. The Einstein manifolds form a natural subclass of the class of quasi-Einstein manifolds. A semi-Riemannian manifold $(M, g), n \geq 3$, is called a quasi-Einstein manifold if at every $x \in M$ its Ricci tensor $S$ has the form

$$
S=\alpha g+\epsilon w \otimes w, \quad \epsilon= \pm 1,
$$

where $w \in T_{x}^{*} M$ and $\alpha \in \mathbb{R}$. For precise definitions of the symbols used we refer to Sections 2 and 3 of [28] (see also [3] and [15]). Another subclass of quasi-Einstein manifolds form Ricci-simple manifolds, i.e. semi-Riemannian manifolds having the Ricci tensor of rank at most one. Quasi-Einstein manifolds arose in the study of exact solutions of the Einstein field equations and in the study of quasi-umbilical hypersurfaces of conformally flat spaces. Quasi-Einstein hypersurfaces were studied among others in [14], [17], and [19], see also references therein. We refer to [3] for a review of results on quasi-Einstein manifolds. We mention that the problem of equivalence of the conditions of semisymmetry

2000 Mathematics Subject Classification: Primary 53B20, 53B25; Secondary 53C25.

Key words and phrases: Roter type hypersurface, Akivis-Goldberg type hypersurface, Cartan type hypersurface, Ricci-pseudosymmetric hypersurface.

Research supported by the Agricultural University of Wrocław (Poland) grant 225/GW/2003 and the VolkswagenStiftung (Germany).

The paper is in final form and no version of it will be published elsewhere. 
$(R \cdot R=0,[27])$ and Ricci-semisymmetry $(R \cdot S=0)$, on hypersurfaces in Euclidean spaces, named the problem of $P$. J. Ryan, leads to consideration of quasi-Einstein hypersurfaces (see e.g. [1], [12] and [18]).

A semi-Riemannian manifold $(M, g), n \geq 3$, is said to be pseudosymmetric ([3], [10]) if, at every point of $M$, the tensors $R \cdot R$ and $Q(g, R)$ are linearly dependent. This is equivalent to

$$
R \cdot R=L_{R} Q(g, R)
$$

on $U_{R}=\left\{x \in M \mid R-\frac{\kappa}{(n-1) n} G \neq 0\right.$ at $\left.x\right\}$, where $L_{R}$ is some function on $U_{R}$. The class of pseudosymmetric manifolds is an extension of the class of semisymmetric manifolds (see e.g. [3], sections 3 and 4). In [25] (see also [26]) a special subclass of pseudosymmetric manifolds was introduced. Namely, according to [25], a pseudosymmetric manifold is called a pseudo-symmetric space of constant type if the function $L_{R}$ is constant.

A semi-Riemannian manifold $(M, g), n \geq 3$, is said to be Ricci-pseudosymmetric ([3], [10]) if, at every point of $M$, the tensors $R \cdot S$ and $Q(g, S)$ are linearly dependent. This is equivalent to

$$
R \cdot S=L_{S} Q(g, S)
$$

on $U_{S}=\left\{x \in M \mid S-\frac{\kappa}{n} g \neq 0\right.$ at $\left.x\right\}$, where $L_{S}$ is some function on $U_{S}$. The class of Ricci-pseudosymmetric manifolds is an extension of the class of Ricci-semisymmetric manifolds, as well as of the class of pseudosymmetric manifolds (see [3] and [10]). A Ricci-pseudosymmetric manifold is called a Ricci-pseudosymmetric manifold of constant type if the function $L_{S}$ is constant. The Cartan hypersurfaces of dimension $\geq 6$ are Ricci-pseudosymmetric manifolds of constant type which are non-pseudosymmetric (see Section 2). Another example of a Ricci-pseudosymmetric manifold of constant type which is non-pseudosymmetric is given in Section 4 of [19].

Let $(M, g), n \geq 4$, be a semi-Riemannian manifold such that its curvature tensor $R$ satisfies on $U_{C} \cap U_{S} \subset M$ the equation

$$
R=\phi \bar{S}+\mu g \wedge S+\eta G,
$$

where $\phi, \mu$ and $\eta$ are some functions on this set and $U_{C}=\{x \in M \mid C \neq 0$ at $x\}$. According to [11], (5) is called the Roter type equation. Consequently, a manifold $(M, g), n \geq 4$, satisfying (5) on $U_{C} \cap U_{S} \subset M$, will be called a Roter type manifold. Obviously, we consider manifolds $(M, g)$ with nonempty set $U_{C} \cap U_{S} \subset M$. We mention that the decomposition of $R$ on $U_{C} \cap U_{S}$ in terms $\bar{S}, g \wedge S$ and $G$ is unique ([17], Lemma 3.2). If (5) holds on an open set $U \subset U_{C} \cap U_{S}$ then we say that the Roter type equation is satisfied on $U$. Roter type manifolds were recently defined in [11], although investigations on these manifolds were initiated earlier in [16]. Examples of such manifolds are presented in [11] and [20].

It is easy to prove that every Roter type manifold is a non-Einstein and non-conformally flat semi-Riemannian manifold of dimension $\geq 4$. The class of Roter type manifolds forms an essential subclass of the class of pseudosymmetric manifolds. Roter type manifolds satisfy also other curvature conditions of pseudosymmetry type (see Section 2). For a survey of results on manifolds satisfying pseudosymmetry type curvature conditions we refer to [3] and [15]. A hypersurface which is a Roter type manifold is called a Roter type 
hypersurface. We can prove that every Roter type manifold satisfies

$$
\begin{aligned}
S \cdot R & =L_{1} \bar{S}+L_{2} g \wedge S+L_{3} G, \\
R \cdot R-Q(S, R) & =L_{4} Q(g, C), \\
S^{2} & =L_{5} S+L_{6} g,
\end{aligned}
$$

where $L_{1}, \ldots, L_{6}$ are some functions on $U_{C} \cap U_{S}$.

According to [11], a semi-Riemannian manifold $(M, g), n \geq 4$, is said to be an AkivisGoldberg type manifold if (6), (7) and (8) hold on $U_{C} \cap U_{S} \subset M$. Every Roter type manifold is an Akivis-Goldberg type manifold. The converse statement is not true ([11]). We refer to [11] for a survey of results on Akivis-Goldberg type manifolds. Again, investigations on these manifolds were initiated in earlier papers: [8] and [23] (see also [2]).

Investigations of curvature properties of Ricci-pseudosymmetric hypersurfaces, and in particular, of the Cartan hypersurfaces of dimension $\geq 6$ (see [13] and references therein), as well as the considerations presented above lead to the definition of the class of Cartan type manifolds. A semi-Riemannian manifold $(M, g), n \geq 4$, is said to be a manifold of Cartan type if on $U_{C} \cap U_{S} \subset M$ we have (7), (8) and

$$
S \cdot R=L_{0} R+L_{1} \bar{S}+L_{2} g \wedge S+L_{3} G,
$$

where $L_{0}, \ldots, L_{6}$ are some functions on $U_{C} \cap U_{S}$. Every Akivis-Goldberg type manifold is a Cartan type manifold. The converse statement is not true (see Theorem 3.4).

Hypersurfaces which are Akivis-Goldberg type manifolds and hypersurfaces which are Cartan type manifolds will be called Akivis-Goldberg type hypersurfaces and Cartan type hypersurfaces, respectively. In Section 3 we prove that every Cartan hypersurface of dimension $\geq 6$ is a Cartan type hypersurface which is not an Akivis-Goldberg type hypersurface.

In [22] (Theorem 1), among other things, it was proved that if the minimal polynomial of the second fundamental tensor $H$ of a hypersurface immersed isometrically in a semiRiemannian space of constant curvature is of second degree then such a hypersurface is a pseudosymmetric manifold. In this paper we improve that result. Namely, we prove that such a hypersurface is a Roter type hypersurface (see Theorem 3.1). As an immediate consequence of Theorem 3.1 we have

THEOREM 1.1. Let $M$ be a hypersurface in a Riemannian space of constant curvature $N^{n+1}(c), n \geq 4$. If at every point of $U_{C} \cap U_{S}$ there are exactly two distinct principal curvatures then $M$ is a Roter type hypersurface.

The converse statement is not true. Namely, we can prove that the hypersurface considered in [17](Example 5.2) is a Roter type hypersurface with three distinct principal curvatures. We mention that quasi-umbilical hypersurfaces of dimension $\geq 4$ in Riemannian spaces of constant curvature are non-Roter type pseudosymmetric hypersurfaces (see Remark 3.1). But on the other hand, every non-Einstein and non-conformally flat Clifford torus of dimension $\geq 4$ is a Roter type hypersurface (see Corollary 3.2). It is well-known that every Clifford torus is a semisymmetric manifold. Furthermore in Example 3.1 we present an example of a warped product Roter type manifold which can be locally realized as a hypersurface in a semi-Euclidean space. 
The author would like to express her thanks to the referee for his hints, remarks and comments.

2. Preliminaries. It is easy to check that (2) implies

$$
S^{2}=(\kappa-(n-2) \alpha) S+\alpha((n-1) \alpha-\kappa) g .
$$

Thus we have

Proposition 2.1. Let $(M, g), n \geq 4$, be a semi-Riemannian manifold satisfying (2). Then (8), with $L_{5}=\kappa-(n-2) \alpha$ and $L_{6}=\alpha((n-1) \alpha-\kappa)$, is satisfied on M. Moreover, if (6) and (7) hold on $U_{C} \cap U_{S} \subset M$ then $(M, g)$ is an Akivis-Goldberg type manifold.

Further, we have

Proposition 2.2. Let $(M, g), n \geq 4$, be a semi-Riemannian manifold. The following conditions: (2) and

$$
\bar{S}=\alpha g \wedge S-\alpha^{2} G=\alpha^{2} G+\alpha \epsilon g \wedge(w \otimes w)
$$

are equivalent on $U_{S} \subset M$.

Proof. Our assertion is an immediate consequence of Lemma 3.1 of [24] and (2).

We can also prove the following

Proposition 2.3. Let $(M, g), n \geq 4$, be a semi-Riemannian manifold satisfying (9). Let $V$ be a set of all points of $U_{C} \cap U_{S} \subset M$ at which (2) or (5) is fulfilled. Then the decomposition of the tensor $S \cdot R$ in terms $R, \bar{S}, g \wedge S$ and $G$ is unique on $\left(U_{C} \cap U_{S}\right) \backslash V$. Proof. Let $x \in\left(U_{C} \cap U_{S}\right) \backslash V$ and let

$$
S \cdot R=L_{0}^{\prime} R+L_{1}^{\prime} \bar{S}+L_{2}^{\prime} g \wedge S+L_{3}^{\prime} G
$$

at $x$, where $L_{0}^{\prime}, \ldots, L_{3}^{\prime} \in \mathbb{R}$. We suppose that at $x$ we have $L_{0}-L_{0}^{\prime} \neq 0$. Now (9) and (11) imply (5), a contradiction. Thus at $x$ we have $L_{0}=L_{0}^{\prime}$. Further, we suppose that at $x$ we have $L_{1}-L_{1}^{\prime} \neq 0$. Now (9) and (11) imply (10). This, in view of Lemma 3.1 [24], turns into (2), a contradiction. Thus at $x$ we have $L_{1}=L_{1}^{\prime}$. Similarly, we prove that $L_{2}=L_{2}^{\prime}$ and $L_{3}=L_{3}^{\prime}$ at $x$. Our proposition is thus proved.

We can check that (5) implies (3) with $L_{R}=(n-2)\left(\frac{\mu}{\phi}\left(\mu-\frac{1}{n-2}\right)-\eta\right)([16]$, Theorem 4.2) and

$$
\begin{gathered}
R \cdot R-Q(S, R)=\left(L_{R}+\frac{\mu}{\phi}\right) Q(g, C), \\
S^{2}=\alpha S+\beta g, \\
S \cdot R=-4(\alpha \phi+\mu) \bar{S}-2(\alpha \mu+\eta+\beta \phi) g \wedge S-4 \beta \mu G, \\
\alpha=\kappa+\frac{(n-2) \mu-1}{\phi}, \quad \beta=\frac{\mu \kappa+(n-1) \eta}{\phi} .
\end{gathered}
$$

Furthermore, we have $C \cdot C=L_{C} Q(g, C)$ and $C \cdot R=L_{C} Q(g, R)$, where $L_{C}=L_{R}+$ $\frac{1}{n-2}\left(\frac{\kappa}{n-1}-\alpha\right)$. We have

Theorem 2.1 ([11], Theorem 3.1). Every Roter type semi-Riemannian manifold $(M, g)$, $n \geq 4$, is an Akivis-Goldberg type manifold. 
Proposition 2.4. On every semi-Riemannian Einstein manifold $(M, g), n \geq 4$, (8) and (9) are satisfied on $M$. Moreover, if $(M, g)$ is pseudosymmetric then (7) holds on $M$.

Proof. Our proposition is an immediate consequence of (11) of [28] and (1), (8) and (9).

From Propositions 2.3 and 2.4 it follows that consideration of Einstein manifolds, as well as of conformally flat manifolds, satisfying (6) (or (9)), (7) and (8) is rather uninteresting.

Using Propositions 2.1 and 2.3 and Theorems 3.3 and 3.4 of [9] we can prove the following

Proposition 2.5. If (7) is satisfied on a conformally flat semi-Riemannian manifold $(M, g), n \geq 4$, then (8) and (9) hold on $M$.

3. Ricci-pseudosymmetric hypersurfaces. Let $M$ be a hypersurface immersed isometrically in a semi-Riemannian manifold $\left(N, g^{N}\right)$. If (5), resp. (6), (7) and (8) or (7), (8) and (9), hold on $U_{C} \cap U_{S} \subset M$ then $M$ is said to be a Roter type hypersurface, resp. an Akivis-Goldberg type hypersurface ([11]) or a Cartan type hypersurface.

Let $N_{s}^{n+1}(c), n \geq 4$, denote a semi-Riemannian space of constant curvature, with signature $(s, n+1-s)$, where $c=\frac{\widetilde{\kappa}}{n(n+1)}$ and $\widetilde{\kappa}$ being its scalar curvature. Further, let $M$ be a hypersurface immersed isometrically in $N_{s}^{n+1}(c)$. The Gauss equation of $M$ in $N_{s}^{n+1}(c)$ reads (see e.g. [15], [28])

$$
R_{h i j k}=\varepsilon\left(H_{h k} H_{i j}-H_{h j} H_{i k}\right)+\frac{\widetilde{\kappa}}{n(n+1)} G_{h i j k}
$$

where $R_{h i j k}, G_{h i j k}$ and $H_{i j}$ denote the local components of the curvature tensor $R$, the tensor $G$ and the second fundamental tensor $H$ of $M$. Contracting (15) with $g^{i j}$ and $g^{k h}$, respectively, we obtain

$$
\begin{aligned}
S_{h k} & =\varepsilon\left(\operatorname{tr}(H) H_{h k}-H_{h k}^{2}\right)+\frac{(n-1) \widetilde{\kappa}}{n(n+1)} g_{h k}, \\
\kappa & =\varepsilon\left((\operatorname{tr}(H))^{2}-\operatorname{tr}\left(H^{2}\right)\right)+\frac{(n-1) \widetilde{\kappa}}{n+1},
\end{aligned}
$$

respectively, where $\kappa$ is the scalar curvature of $M, \operatorname{tr}(H)=g^{h k} H_{h k}, \operatorname{tr}\left(H^{2}\right)=g^{h k} H_{h k}^{2}$ and $S_{h k}$ are the local components of the Ricci tensor $S$ of $M$. For the definition of the tensor $H^{2}$ see e.g. [28] (Section 1). Further, we denote by $U_{H}$ the set of all points of $M$ at which the tensor $H^{2}$ is not a linear combination of the metric tensor $g$ and the second fundamental tensor $H$ of $M$. Using (16) and Theorem 4.1 of [21] we can deduce that $U_{H} \subset U_{C} \cap U_{S} \subset M$. Evidently, on $\left(U_{C} \cap U_{S}\right) \backslash U_{H}$ we have

$$
H^{2}=\alpha H+\beta g
$$

where $\alpha$ and $\beta$ are some functions on $\left(U_{C} \cap U_{S}\right) \backslash U_{H}$.

We recall that a Cartan hypersurface in the sphere $S^{n+1}(c)$ is a compact minimal hypersurface with constant principal curvatures $-(3 c)^{\frac{1}{2}}, 0,(3 c)^{\frac{1}{2}}$ of the same multiplicity $\frac{n}{3}$. It is known that the Cartan hypersurfaces are tubes of constant radius over the standard Veronese embeddings $i: \mathbb{E} P^{2} \rightarrow S^{3 d+1}(c) \rightarrow \mathbb{E}^{3 d+2}, d=1,2,4,8$, of the projective plane $\mathbb{E} P^{2}$ into the sphere $S^{3 d+1}(c)$ in a Euclidean space $\mathbb{E}^{3 d+2}$, where $\mathbb{E}=\mathbb{R}$ (real numbers), 
$\mathbb{C}$ (complex numbers), $\mathbb{Q}$ (quaternions) or $\mathbb{O}$ (Cayley numbers), respectively ([4]). Every Cartan hypersurface satisfies (4). Precisely, we have (see e.g. [13], Theorem 4.3)

$$
R \cdot S=\frac{\widetilde{\kappa}}{n(n+1)} Q(g, S)
$$

Thus such a hypersurface is a Ricci-pseudosymmetric manifold of constant type. In addition, the Cartan hypersurface in $S^{4}(c)$ is a pseudosymmetric manifold satisfying ([22], Example 2)

$$
R \cdot R=\frac{\widetilde{\kappa}}{12} Q(g, R) .
$$

If on the set $U_{H}$ of a hypersurface $M$ in $N_{s}^{n+1}(c), n \geq 3$, we have $H^{3}=\operatorname{tr}(H) H^{2}+\lambda H$, for some function $\lambda$, then (18) holds on $U_{H}$ ([7], Proposition 3.2). It is also known that if $\operatorname{rank} H=2$ at every point of a hypersurface $M$ in $N_{s}^{n+1}(c), n \geq 3$, then it is a pseudosymmetric space of constant type ([7], Theorem 4.2). Precisely, on such a hypersurface we have

$$
R \cdot R=\frac{\widetilde{\kappa}}{n(n+1)} Q(g, R)
$$

On every hypersurface $M$ in $N_{s}^{n+1}(c), n \geq 4$, we have ([21])

$$
R \cdot R-Q(S, R)=-\frac{(n-2) \widetilde{\kappa}}{n(n+1)} Q(g, C) .
$$

Thus on $U_{C} \cap U_{S} \subset M$ we have (8) with $L_{4}=-\frac{(n-2) \widetilde{\kappa}}{n(n+1)}$. Clearly, if (6) and (8), resp. (8) and (9), hold on $U_{C} \cap U_{S} \subset M$ then $M$ is an Akivis-Goldberg type hypersurface, resp. a Cartan type hypersurface.

Theorem 4.3 of [13] states that every Cartan hypersurface of dimension $n \geq 6$ is a Cartan type hypersurfce. They are non-pseudosymmetric Ricci-pseudosymmetric hypersurfaces. Further, (9), with $L_{1}=0$, is satisfied on $U_{H} \subset U_{C} \cap U_{S} \subset M$ for every Ricci-pseudosymmetric hypersurface $M$ in $N_{s}^{n+1}(c), n \geq 4$, ([13], Theorem 3.2). In other words, on $U_{H}$ we have (8) and

$$
S \cdot R=L_{0} R+L_{2} g \wedge S+L_{3} G .
$$

In this section we prove that such hypersurfaces are Cartan type hypersurfaces. We also mention that examples of quasi-Einstein Ricci-pseudosymmetric hypersurfaces were found recently in [17] and [19].

Since (19) is a relation of the form (7), we have

Proposition 3.1. Let $M$ be a hypersurface in $N_{s}^{n+1}(c), n \geq 4$.

(i) The relation (7) is satisfied on $M$.

(ii) If (6) and (8) hold on $U_{C} \cap U_{S} \subset M$ then $M$ is an Akivis-Goldberg type hypersurface.

Propositions 2.1 and 3.1 imply

Proposition 3.2. If $M$ is a hypersurface in $N_{s}^{n+1}(c), n \geq 4$, satisfying (2) and (6) on $U_{C} \cap U_{S} \subset M$ then $M$ is an Akivis-Goldberg type hypersurface. 
Proposition 3.3. Let $M$ be a hypersurface in $N_{s}^{n+1}(c), n \geq 4$. Then on $\left(U_{C} \cap U_{S}\right) \backslash U_{H} \subset$ $M$ we have

$$
\begin{aligned}
R= & \varepsilon(\operatorname{tr}(H)-\alpha)^{-2}\left(\bar{S}-\left(\frac{(n-1) \widetilde{\kappa}}{n(n+1)}-\varepsilon \beta\right) g \wedge S\right) \\
& +\left(\varepsilon(\operatorname{tr}(H)-\alpha)^{-2}\left(\frac{(n-1) \widetilde{\kappa}}{n(n+1)}-\varepsilon \beta\right)^{2}+\frac{\widetilde{\kappa}}{n(n+1)}\right) G,
\end{aligned}
$$

where $\alpha$ and $\beta$ are defined by (17).

Proof. First of all, we note that (16) and (17) yield

$$
S=\varepsilon(\operatorname{tr}(H)-\alpha) H+\left(\frac{(n-1) \widetilde{\kappa}}{n(n+1)}-\varepsilon \beta\right) g .
$$

Thus we see that $\operatorname{tr}(H)-\alpha \neq 0$ at every point of $\left(U_{C} \cap U_{S}\right) \backslash U_{H}$. Further, using (15) and (21) we get (20), completing the proof.

Proposition 3.3 implies

THEOREM 3.1. Let $M$ be a hypersurface in $N_{s}^{n+1}(c), n \geq 4$, and let $\left(U_{C} \cap U_{S}\right) \backslash U_{H} \subset M$ be nonempty. Then the Roter type equation holds on this set. Moreover, if $U_{H} \subset M$ is empty then $M$ is a Roter type hypersurface.

It is easy to see that the last theorem implies Theorem 1.1.

REMARK 3.1. Let $M$ be a hypersurface in a Riemannian space of constant curvature $N^{n+1}(c), n \geq 4$. Using the well-known Cartan-Schouten result (see e.g. [5], Section 13.1.1.) we can easily deduce that if at a point of $U_{C} \subset M$ there are exactly two distinct principal curvatures then the multiplicity of each principal curvature is $\geq 2$, i.e. $M$ cannot be quasi-umbilical at this point. More generally, if $M$ is a hypersurface in $N_{s}^{n+1}(c)$, $n \geq 4$, and if (17) is satisfied at a point of $U_{C} \subset M$ then $M$ is not quasi-umbilical at this point ([21], Theorem 4.1).

TheOREM 3.2 ([13], Theorem 3.2). If $M$ is a Ricci-pseudosymmetric hypersurface in $N_{s}^{n+1}(c), n \geq 4$, then (8) and (9) hold on $U_{H} \subset M$.

Theorems 3.1 and 3.2 yield

ThEOREM 3.3. Every Ricci-pseudosymmetric hypersurface in $N_{s}^{n+1}(c), n \geq 4$, is a Cartan type hypersurface.

THEOREM 3.4. The Cartan hypersurface $M$ in $S^{n+1}(c), n=6,12$ or 24 is a Cartan type hypersurface which is not an Akivis-Goldberg type hypersurface.

Proof. First of all we note that $M$ is a non-pseudosymmetric Ricci-pseudosymmetric hypersurface (e.g. see [13], section 2). From the definition of the Cartan hypersurface it follows that $U_{C} \cap U_{S}=M$. We suppose now that $M$ is an Akivis-Goldberg type hypersurface. Thus on $M$ we have $S \cdot R=L_{1}^{\prime} \bar{S}+L_{2}^{\prime} g \wedge S+L_{3}^{\prime} G$, where $L_{1}^{\prime}, L_{2}^{\prime}$ and $L_{3}^{\prime}$ are some functions on $M$. The last relation, together with the equation (53) of [13], yields (5). But (5) implies (3) (see section 2), a contradiction. Thus our theorem is proved.

EXAmple 3.1. Let $M_{1}$, resp. $M_{2}$, be a nonempty open connected subset of $\mathbb{R}^{p}$, resp. $\mathbb{R}^{n-p}, 2 \leq p \leq n-2$, equipped with the standard metric $g_{1}, g_{1 a b}=\varepsilon_{a} \delta_{a b}, \varepsilon_{a}= \pm 1$, resp. 
$g_{2}, g_{2 \alpha \beta}=\varepsilon_{\alpha} \delta_{\alpha \beta}, \varepsilon_{\alpha}= \pm 1$, where $a, b, c, d, e, f \in\{1, \ldots, p\}, \alpha, \beta, \gamma, \delta \in\{p+1, \ldots, n\}$ and $h, i, j, k \in\{1, \ldots, n\}$. We set $F=F\left(x^{1}, \ldots, x^{p}\right)=k \exp \left(\xi_{a} x^{a}\right)$, where $k, \xi_{1}, \ldots, \xi_{p} \in \mathbb{R}$, $\xi_{1}^{2}+\ldots+\xi_{p}^{2}>0, k>0$ and $g_{1}{ }^{e f} \xi_{e} \xi_{f} \neq 0$. We consider the warped product $M_{1} \times_{F} M_{2}$. Let $\tau$ be a function on $\bar{M} \times_{F} \widetilde{N}$ defined by (cf. [8], Section 4)

$$
\tau^{2}=-\frac{\varepsilon}{4} g_{1}{ }^{e f} \xi_{e} \xi_{f}, \quad \varepsilon= \pm 1 .
$$

It is clear that there exist constants $\varepsilon, \varepsilon_{a}$, and $\xi_{a}$ such that the right-hand side of (22) is positive at every point $x$ of $\bar{M} \times_{F} \tilde{N}$. Using now (44) of [8] and (22) we find

$$
\begin{aligned}
S_{a b} & =-\frac{n-p}{4} \xi_{a} \xi_{b}, \quad S_{\alpha \beta}=(n-p) \varepsilon \tau^{2} g_{\alpha \beta}, \\
S^{2} & =\frac{\kappa}{n-p+1} S, \quad \kappa=(n-p)(n-p+1) \varepsilon \tau^{2} .
\end{aligned}
$$

In Section 4 of [8] it was shown that the conditions: $R \cdot R=Q(S, R)$ and $S \cdot R=0$ are satisfied on $\bar{M} \times_{F} \widetilde{N}$. Further, we have (see [8], section 4) $R=\varepsilon \bar{H}$, where $H$ is a symmetric $(0,2)$-tensor with the local components

$$
H_{a b}=-\frac{1}{4 \tau} \xi_{a} \xi_{b}, \quad H_{a \alpha}=0, \quad H_{\alpha \beta}=\varepsilon \tau g_{\alpha \beta}
$$

The tensor $H$ is a Codazzi tensor. Therefore $M_{1} \times_{F} M_{2}$ locally can be realized as a hypersurface in a semi-Euclidean space $\mathbb{E}_{s}^{n+1}$. From (23) and (24) it follows that

$$
\operatorname{tr} H=-\frac{1}{4 \tau} g^{1 e f} \xi_{e} \xi_{f}+(n-p) \varepsilon \tau=(n-p+1) \varepsilon \tau, \quad H^{2}=\varepsilon \tau H .
$$

Applying this and (23) into (16) and (15), on $M_{1} \times_{F} M_{2}$ we get

$$
S=(n-p) \tau H, \quad R=\frac{n-p+1}{(n-p) \kappa} \bar{S},
$$

respectively. Thus we see that $M_{1} \times_{F} M_{2}$ is a Roter type manifold which locally can be realized as a hypersurface in a semi-Euclidean space $\mathbb{E}_{s}^{n+1}$.

REMARK 3.2. (i) An example of a hypersurface $M$ in $N_{s}^{n+1}(c), c \neq 0, n \geq 4$, satisfying the Roter type equation on the set $\left(U_{C} \cap U_{S}\right) \backslash U_{H} \subset M$, is given in [20]. We also mention that warped products satisfying (5) were investigated in [20].

(ii) The warped product defined in Example 4.1 of [14] is a Ricci-simple Akivis-Goldberg type manifold. Clearly, such manifold is not a Roter type manifold. That warped product locally can be realized as a hypersurface in a semi-Euclidean space $\mathbb{E}_{s}^{n+1}$ ([14], Example 5.1).

(iii) Examples of Ricci-pseudosymmetric quasi-Einstein hypersurfaces in spaces of constant curvature are given in [17] and [19].

We consider the Cartesian product $N_{s_{1}}^{p}\left(c_{1}\right) \times N_{s_{2}}^{n-p}\left(c_{2}\right)$ of two semi-Riemannian spaces of constant curvature $N_{s_{1}}^{p}\left(c_{1}\right)$ and $N_{s_{2}}^{n-p}\left(c_{2}\right), 2 \leq p \leq n-2$, where $c_{1}=\frac{\kappa_{1}}{(p-1) p}$, $c_{2}=\frac{\kappa_{2}}{(n-p-1)(n-p)}$ and $\kappa_{1}$ and $\kappa_{2}$ are the scalar curvatures of $N_{s_{1}}^{p}\left(c_{1}\right)$ and $N_{s_{2}}^{n-p}\left(c_{2}\right)$, respectively. This product is a semisymmetric manifold ([27], Theorem 4.5). Further, it is known that the local components $R_{h i j k}$ of the curvature tensor and the local components $S_{h k}$ of the Ricci tensor $S$ of $N_{s_{1}}^{p}\left(c_{1}\right) \times N_{s_{2}}^{n-p}\left(c_{2}\right)$ which might not vanish identically, are 
the following:

$$
\begin{aligned}
R_{a b c d} & =c_{1}\left(g_{a d} g_{b c}-g_{a c} g_{b d}\right), \\
R_{\alpha \beta \gamma \delta} & =c_{2}\left(g_{\alpha \delta} g_{\beta \gamma}-g_{\alpha \gamma} g_{\beta \delta}\right), \\
S_{a d} & =(p-1) c_{1} g_{a d}, \\
S_{\alpha \delta} & =(n-p-1) c_{2} g_{\alpha \delta},
\end{aligned}
$$

where $g_{h k}$ are the local components of the metric tensor of $N_{s_{1}}^{p}\left(c_{1}\right) \times N_{s_{2}}^{n-p}\left(c_{2}\right), a, b, c, d \in$ $\{1,2, \ldots, p\}$ and $\alpha, \beta, \gamma, \delta \in\{p+1, p+2, \ldots, n\}$. The scalar curvature $\kappa$ of $N_{s_{1}}^{p}\left(c_{1}\right) \times$ $N_{s_{2}}^{n-p}\left(c_{2}\right)$ is given by

$$
\kappa=p(p-1) c_{1}+(n-p)(n-p-1) c_{2} .
$$

In addition, we assume that the considered product satisfies

$$
\text { (a) } c_{1}+c_{2} \neq 0 \text { and } \quad(b) \quad(p-1) c_{1} \neq(n-p-1) c_{2} \text {. }
$$

Using (25) and (27) we can conclude that $N_{s_{1}}^{p}\left(c_{1}\right) \times N_{s_{2}}^{n-p}\left(c_{2}\right), 2 \leq p \leq n-2$, is a non-conformally flat and non-Einstein manifold. Further, from (25) it follows that also (5) is satisfied with

$$
\begin{aligned}
\phi & =\tau\left(c_{1}+c_{2}\right), \\
\mu & =-(n-2) \tau c_{1} c_{2}, \\
\eta & =\tau c_{1} c_{2}\left((p-1)^{2} c_{1}+(n-p-1)^{2} c_{2}\right), \\
\tau & =\left((p-1) c_{1}-(n-p-1) c_{2}\right)^{-2} .
\end{aligned}
$$

Moreover, (26) and (27) yield

$$
\frac{\phi \kappa}{n-1}+\mu-\frac{1}{n-2}=\frac{(p-1)(n-p-1)}{(n-2)(n-1)} \tau\left(c_{1}+c_{2}\right)^{2} .
$$

The above considerations yield

Proposition 3.4. The Cartesian product $N_{s_{1}}^{p}\left(c_{1}\right) \times N_{s_{2}}^{n-p}\left(c_{2}\right), 2 \leq p \leq n-2$, is a Roter type manifold, provided that (27) is satisfied.

EXAMPLE 3.2. Let $S^{p}(r)$ be $p$-dimensional standard sphere of radius $r$ in $\mathbb{E}^{p+1}$. We note that $r^{-2}=\frac{\kappa}{(p-1) p}$, where $\kappa$ is the scalar curvature of the given sphere. From the above considerations it follows that the Cartesian product $S^{p}\left(r_{1}\right) \times S^{n-p}\left(r_{2}\right), 2 \leq p \leq n-2$, of two spheres $S^{p}\left(r_{1}\right)$ and $S^{n-p}\left(r_{2}\right)$ is a Roter type manifold, provided that $(27)(\mathrm{b})$, or equivalently, $(n-p-1) r_{1}^{2} \neq(p-1) r_{2}^{2}$ is satisfied. From Theorem 5.1 of [30] (cf. [6]) it follows that $M=S^{p}\left(r_{1}\right) \times S^{n-p}\left(r_{2}\right), r_{1}=\sqrt{\frac{p}{n}}, r_{2}=\sqrt{\frac{n-p}{n}}, 2 \leq p \leq n-2$, can be realized as a minimal hypersurface immersed isometrically in the sphere $S^{n+1}(1)$ having at every point exactly two distinct principal curvatures $\rho_{1}$ and $\rho_{2}$ of multiplicity $p$ and $n-p$, respectively. It is known that $\rho_{1} \rho_{2}+1=0$ and $\rho_{i}^{2}=r_{i}^{-2}-1, i=1,2$. The hypersurface $M$ is called the Clifford torus.

Corollary 3.1. The Clifford torus $S^{p}\left(\sqrt{\frac{p}{n}}\right) \times S^{n-p}\left(\sqrt{\frac{n-p}{n}}\right), 2 \leq p \leq n-2, n \neq 2 p$, is a Roter type hypersurface.

We finish this section with the following 
REMARK 3.3. Let $(M, g), n \geq 4$, be a semi-Riemannian manifold satisfying

$$
C=\frac{L}{2}\left(S-\frac{\kappa}{n-1} g\right) \wedge\left(S-\frac{\kappa}{n-1} g\right)
$$

on $U_{C} \cap U_{S} \subset M$, where $L$ is some function on $U_{C} \cap U_{S}$. Manifolds satisfying (29) were investigated in [24]. Evidently, (29) is equivalent to (5) with $\phi=L, \mu=\frac{1}{n-2}-\frac{L \kappa}{n-1}$ and $\eta=\frac{\kappa}{n-1}\left(\frac{L \kappa}{n-1}-\frac{1}{n-2}\right)$. It is easy to see that on $U_{C} \cap U_{S}$ we have $\frac{\phi \kappa}{n-1}+\mu-\frac{1}{n-2}=0$. Comparing this with (28) we can conclude that a semi-Riemannian manifold $(M, g)$, $n \geq 4$, satisfying (29) on $U_{C} \cap U_{S} \subset M$, and $N_{s_{1}}^{p}\left(c_{1}\right) \times N_{s_{2}}^{n-p}\left(c_{2}\right), 2 \leq p \leq n-2$, satisfying (27), are not isometric.

\section{References}

[1] B. E. Abdalla and F. Dillen, A Ricci-semi-symmetric hypersurface of the Euclidean space which is not semi-symmetric, Proc. Amer. Math. Soc. 130 (2002), 1805-1808.

[2] M. A. Akivis and V. V. Goldberg, Semiintegrable almost Grassmann structures, Differential Geom. Appl. 10 (1999), 257-294.

[3] M. Belkhelfa, R. Deszcz, M. Głogowska, M. Hotloś, D. Kowalczyk, and L. Verstraelen, On some type of curvature conditions, in: Banach Center Publ. 57, Inst. Math. Polish Acad. Sci., 2002, 179-194.

[4] T. E. Cecil and P. J. Ryan, Tight and Taut Immersions of Manifolds, Pitman Publ. Inc., Boston, London, Melbourne, 1988.

[5] B. Y. Chen, Riemannian submanifolds, Handbook of Differential Geometry (eds. F. Dillen and L. Verstraelen), vol. 1, North Holland, Amsterdam, 2000, 187-418.

[6] S. S. Chern, M. do Carmo, and S. Kobayashi, Minimal submanifolds of a sphere with second fundamental form of constant length, in: Functional Analysis and Related Fields, Springer, 1970, 59-75.

[7] F. Defever, R. Deszcz, P. Dhooghe, L. Verstraelen, and S.. Yaprak, On Riccipseudosymmetric hypersurfaces in spaces of constant curvature, Results Math. 27 (1995), $227-236$.

[8] F. Defever, R. Deszcz, M. Głogowska, V. V. Goldberg, and L. Verstraelen, A class of four-dimensional warped products, Demonstr. Math. 37 (2002), 853-864.

[9] R. Deszcz, On conformally flat Riemannian manifolds satisfying certain curvature conditions, Tensor, (N.S.), 49 (1990), 134-145.

[10] R. Deszcz, On pseudosymmetric spaces, Bull. Soc. Belg. Math., Ser. A 44 (1992), 1-34.

[11] R. Deszcz, On some Akivis-Goldberg type metrics, Publ. Inst. Math. (Beograd) (N.S.) 74 (84) (2003), 71- 83.

[12] R. Deszcz and M. Głogowska, Examples of nonsemisymmetric Ricci-semisymmetric hypersurfaces, Colloq. Math. 94 (2002), 87-101.

[13] R. Deszcz and M. Głogowska, Some nonsemisymmetric Ricci-semisymmetric warped product hypersurfaces, Publ. Inst. Math. (Beograd) (N.S.) 72(86) 2002, 81-93.

[14] R. Deszcz, M. Głogowska, M. Hotloś, and Z. Șentürk, On certain quasi-Einstein semisymmetric hypersurfaces, Ann. Univ. Sci. Budapest. Eötvös Sect. Math. 41 (1998), 151-164.

[15] R. Deszcz, M. Głogowska, M. Hotloś, and L. Verstraelen, On some generalized Einstein metric conditions on hypersurfaces in semi-Riemannian space forms, Colloq. Math. 96 (2003), 149-166. 
[16] R. Deszcz and M. Hotloś, On a certain subclass of pseudosymmetric manifolds, Publ. Math. Debrecen 53 (1998), 29-48.

[17] R. Deszcz and M. Hotloś, On hypersurfaces with type number two in space forms, Ann. Univ. Sci. Budapest. Eötvös Sect. Math. 46 (2003), 19-34.

[18] R. Deszcz, M. Hotloś, and Z. Șentürk, On curvature properties of quasi-Einstein hypersurfaces in semi-Euclidean spaces, Soochow J. Math. 27 (2001), 375-389.

[19] R. Deszcz, M. Hotloś, and Z. Șentürk, On curvature properties of certain quasi-Einstein hypersurfaces, Dept. Math., Agricultural Univ. Wrocław, Ser. A, Theory and Methods, Report No. 104, 2002.

[20] R. Deszcz and D. Kowalczyk, On some class of pseudosymmetric warped products, Colloq. Math. 97 (2003), 7-22.

[21] R. Deszcz and L. Verstraelen, Hypersurfaces of semi-Riemannian conformally flat manifolds, in: Geometry and Topology of Submanifolds, III, World Sci., River Edge, NJ, 1991, 131-147.

[22] R. Deszcz, L. Verstraelen and Ș. Yaprak, Pseudosymmetric hypersurfaces in 4-dimensional spaces of constant curvature, Bull. Inst. Math. Acad. Sinica 22 (1994), 167-179.

[23] M. Głogowska, Curvature properties of some four-dimensional manifolds, Demonstr. Math. 34 (2001), 901-918.

[24] M. Głogowska, Semi-Riemannian manifolds whose Weyl tensor is a Kulkarni-Nomizu square, Publ. Inst. Math. (Beograd) (N.S.) 72(86) 2002, 95-106.

[25] O. Kowalski and M. Sekizawa, Pseudo-symmetric spaces of constant type in dimension three-elliptic spaces, Rend. Mat. Appl. (7) 17 (1997), 477-512.

[26] O. Kowalski and M. Sekizawa, Pseudo-symmetric spaces of constant type in dimension three—non-elliptic spaces, Bull. Tokyo Gakugei Univ., Sect. IV, Math. Nat. Sci. 50 (1998), $1-28$.

[27] Z. I. Szabó, Structure theorems on Riemannian spaces satisfying $R(X, Y) \cdot R=0$. I. The local version, J. Differential Geom. 17 (1982), 531-582.

[28] K. Sawicz, On some class of hypersurfaces with three distinct principal curvatures, this volume, $145-156$.

[29] F. Tricerri and L. Vanhecke, Cartan hypersurfaces and reflections, Nihonkai Math. J. 1 (1990), 203-208.

[30] K. Yano and S. Ishihara, Submanifolds with parallel mean curvature vector, J. Differential Geom. 6 (1971), 95-118. 